\title{
Air curtains of open refrigerated display cases revisited: a new technique for infiltration rate measurements
}

\author{
M. Amin ${ }^{1}$, H. K. Navaz ${ }^{2}$, D. Dabiri ${ }^{1}$ \& R. Faramarzi ${ }^{3}$ \\ ${ }^{1}$ Aeronautics and Astronautics Engineering Department, \\ University of Washington, WA, USA \\ ${ }^{2}$ Mechanical Engineering Department, Kettering University, MI, USA \\ ${ }^{3}$ Refrigeration and Thermal Test Center, \\ Southern California Edison Company, CA, USA
}

\begin{abstract}
Air curtains are created in open refrigerated vertical display cases for creating an invisible barrier between the cold air inside and the warm air outside the case. A systematic approach is developed to minimize the entrainment and infiltration of warm air into the case by optimizing the performance of an air curtain through adjusting pertinent flow parameters and case geometry. A modular display case was manufactured for the parametric studies. In this modular display case the geometry and flow parameters were changed and the infiltration rate was measured using a new technique, tracer gas method, which could be performed noticeably faster than the conventional methods. This apparatus is referred to as the proof-of-concept air curtain (POCAC). A matrix of all possible permutations was constructed with the infiltration rate being the outcome of this matrix. This matrix was populated by actual experimental measurements as well as using validated Computational Fluid Dynamics (CFD) computer programs as a tool to acquire better resolution of the input and output datasets. All the problem variables are referred to as the input vector of all parameters that can be altered, and the output is the infiltration rate. An artificial neural network (ANN) program was used to provide the linkage between the input vector (problem variables) and the problem outcome (infiltration). This program can also be used by industry as a tool to estimate the infiltration rate for all existing open vertical display cases.
\end{abstract}

Keywords: air curtain, open vertical refrigerated display case, infiltration, entrainment, tracer gas, neural network, experiment, CFD, neural network. 


\section{Nomenclature}

A a constant

$B$ a constant

CFD Computational Fluid Dynamics

$C$ mass concentration of tracer gas $\left(\frac{\dot{m}_{\text {trac }}}{\dot{m}_{\text {trac }}+\dot{m}_{\text {air }}}\right)$

$D \quad$ average distance between the shelves

$D A G$ Discharge Air Grille

$G r \quad$ Grashof number $\left(\frac{G \beta_{\circ}\left(T_{D A G}-T_{r}\right) H^{3}}{v}\right)$

$H \quad$ opening height

$I \quad$ average turbulence intensity at the $D A G$

$L \quad$ length of the display case in z-direction

$\dot{m}$ mass flow rate

N.I.R. Non-dimensional Infiltration Rate

$R A G$ Return Air Grille

Re Reynolds number $(V w / v)$

R.H. Relative Humidity

$R i \quad$ Richardson number $(\mathrm{Gr} / \mathrm{Re})$

$T$ average temperature

$V \quad$ average normal velocity

$w \quad$ width

$X \quad$ average horizontal distance between perforated back panel and $D A G$

$Y \quad$ horizontal offset distance between $D A G$ and RAG

Greek symbols

$\alpha \quad$ offset angle $\alpha=\tan ^{-1}\left(\frac{Y}{H}\right)$

$\eta \quad$ thermal entrainment

$\beta \quad$ throw angle

$\beta$ 。 thermal expansion coefficient

$v \quad$ kinematic viscosity

Subscripts:

$B P \quad$ Back Panel

$D A G$ Discharge Air Grille

inf infiltrated

$R m$ room

$R A G$ Return Air Grille

$s \quad$ shelf

tot total flow rate

trac tracer gas

\section{Introduction}

An air curtain is a planar jet of air with large aspect ratio that has higher momentum than its surrounding air and is mainly responsible for separating two 
adjacent zones that have different characteristics and properties, e.g. temperature, airborne particle, relative humidity, etc. There are various applications for creating an air curtain such as infant incubators and clean rooms in hospitals, thermal protection of storage rooms and many aspects of the HVAC industry, microelectronics production line, managing fire in tunnels, protecting art work against airborne pollutions, preventing the penetration of warm air into open refrigerated display cases (current work), etc. Air curtains are usually introduced in vertical directions and depending on the application, they may be circulated through the system (current work), or impinged on a surface located in the downstream of the jet. They may also be isothermal or thermally conditioned depending on the specific application. This work focuses on the protection of the food and products on the shelves in an open refrigerated display case schematically shown in Figure 1, and prevents the penetration of the warmer room air into the cold conditioned display case air.

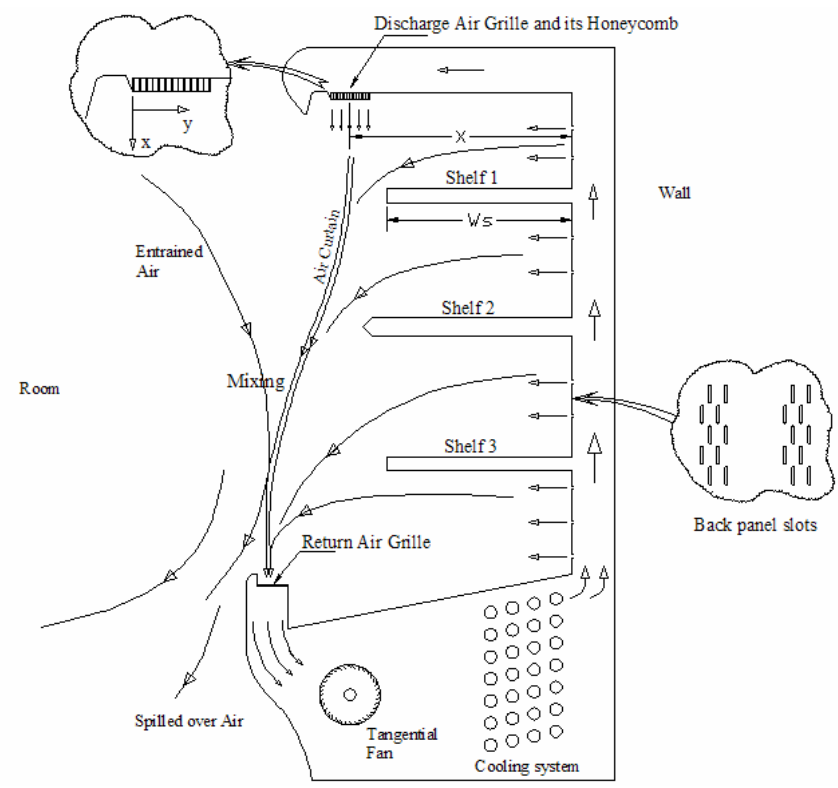

Figure 1: Schematic of a typical display case without food.

The penetration is initiated by entrainment mechanism of the room air due to shear effects into the air curtain. The entrained air with higher content of energy than the supplied air by display case, proceeds in two directions: a portion of that reaches the shelves' products and directly increases their temperature and the rest moves towards the Return Air Grille (RAG), through which the air is circulated. The circulated air that is a mixture of cold and warm air is reconditioned by passing it through the cooling coils of the display case causing the major portion of the cooling load.

One of the early studies on air curtains was performed by Howell et al. [1]. Their work showed that heat and mass transfer through air curtains directly 
depends on the velocity of air at the discharge air grille $(D A G)$. It should be noted that the air curtain is mainly momentum driven causing some shear with the neighboring warm air therefore resulting in mixing. To reduce the amount of mixing, it is important for the air stream to maintain its integrity and path implying that sufficient momentum should exist at the origination point. However, increasing the velocity at the $D A G$ will enhance turbulence in the air curtain path as it moves down towards the $R A G$. This increase in turbulence intensity will induce more mixing with the warm air along the main path of the flow, thereby increasing the infiltration rate. In his other work Howell and Shiabata [2] showed that the ratio of the opening height to the width of the $D A G$ influences the performance of the air curtains. For a given velocity, the excessive increase of the opening height will cause the air curtain to bend outward and lose its structure and break into a wide region of mixing layers before approaching the $R A G$. On the other hand reducing the opening height will have some practical implications by constraining food accessibility. Howell and Shiabata [2] and Nuygen et al [3] have performed some preliminary numerical studies with turbulence intensity at a free jet $D A G$ and its effect on non-circulated air curtains. However, a jet with a circulating air return that can be situated in different locations (in front of the jet or with an offset distance from the jet exit plane) is a more complex problem. The additional complexity can be induced by the geometrical factors, i.e. a free jet is not a bounded domain, while the geometry in a display case resembles a cavity (Rouaud and Havet, [4]). Furthermore, for a non-isothermal flow the air curtain is subject to a transverse pressure difference caused by stack effect (Hayes and Stoecker [5]). Further studies by Howell and Adams [6] revealed that up to $75 \%$ of the refrigeration load of display cases with circulated air curtains could be attributed to the entrainment and infiltration from the room air. In terms of non-dimensional quantities, the above parameters can be grouped as $V w_{D A G} / v$ (Reynolds number), $I_{D A G} / V$, and $H / w_{D A G}$. Some of the recent studies have focused mostly on the optimization of air curtains in open refrigerated display cases. Several factors such as velocity magnitude, velocity profile shape, and turbulence intensity all at the $D A G$, and some geometrical dimensions of the system including opening height, the horizontal position of the $R A G$ relative to the $D A G$, the ratio of the discharged flow through the air curtain to that of the perforated back panel, etc. can affect the performance of the air curtains. Some of these factors have been addressed in the work of many researches, but almost none has investigated the problem in a systematic approach. During the late 90's CFD applications found their way into analyzing mainly the air flow, heating and/or cooling in food industry [7]. A more modern analytical approach that takes advantage of sophisticated tools such as CFD with the same basic goal has been adopted by Axell and Fahlen $[8,9]$. They developed a correlation for the Nusselt Number of air curtain that related to the amount of heat transfer and cooling load thereafter. Navaz et al. [10] have demonstrated that a marriage between the Digital Particle Image Velocimetry (DPIV) and CFD simulation can be quite effective in developing a milestone for systematic study of air curtains. They have implemented the boundary conditions taken from the experimental results and demonstrated that curve fits similar to previous works 
can be re-produced. Then they used their hybrid CFD/DPIV approach to identify the most important parameters that can impact the performance of an air curtain [11]. They have shown that this hybrid approach can effectively quantify the effect of individual flow/geometrical parameters on the air curtain performance. They have also mentioned that certain operating conditions can result in improved performance of an air curtain [12,13]. Field et al. [14,15] studied circulating air curtains of a display case by the PIV visualization. For better understanding of the buoyancy effect, a flat vertical wall was installed in front of the shelves. They found out that for an isothermal air curtain, the thickness of the jet grows almost linearly downstream and it is somewhat independent of the typical Reynolds numbers. In non-isothermal condition, the negative buoyancy accelerated the jet at low $R i$ values; and this higher momentum causes the spreading of the jet to be delayed. Due to the acceleration, the thickness of the refrigerated curtains was smaller than its isothermal counterpart. Although their work is valuable, they have not considered the effect of the back panel flow, the presence of shelves, and other geometrical factors. The flat wall geometry in their analysis is based on their assumption of the display case to be $100 \%$ filled with food products. Chen and Yuaa [16] and Mhiri et al. [17] numerically studied the effect of the Richardson number on the temperature distribution inside cavities exposed to non-circulated air curtains. Chen et al have discussed that for a given Grashof number there is a Richardson number that must be less than a critical value to assure thermal insulation.

Insofar, there are studies focused on different aspects of the air curtain problem. However, to this date there is no systematic approach that can solve industry problems in answering a fundamental question: Under what flow/geometrical parameters an air curtain performance can be optimized, i.e. minimizing the infiltration rate. It is the main purpose of this paper to demonstrate that a hybrid approach of numerical/experimental/ANN can be effectively used to develop a tool for estimating the infiltration rate of the existing display cases, and also find those optimum conditions that can minimize the infiltration rate with constraints imposed by the manufacturer.

\section{New approach}

In order to achieve the goal of this study that is using a systematic approach to find the minimum infiltration rate in an open refrigerated display case, the most important variables that are shared among all display cases are identified. Basically, all the variables can be divided into two groups, geometric and flow related parameters. By using dimensional analysis the infiltrated mass flow rate can be found to be:

$$
\frac{\dot{m}_{\mathrm{inf}}}{\dot{m}_{t o t}}=f\left(\frac{L}{w_{D A G}}, \frac{H}{w_{D A G}}, \frac{w_{R A G}}{w_{D A G}}, \frac{w_{s}}{w_{D A G}}, \frac{X}{w_{D A G}}, \alpha, \beta, \operatorname{Re}, R H, \frac{D}{w_{D A G}}, \frac{T_{D A G}}{T_{R m}}, \frac{\dot{m}_{B P}}{\dot{m}_{t o t}}\right) \text {. }
$$

where $\dot{m}_{t o t}=\dot{m}_{R A G}=\dot{m}_{D A G}+\dot{m}_{B P}$.

These parameters are depicted in Figures 1 and 2. In eqn (2) considering that the actual data will eventually be required to be taken for all prescribed number 
of levels specified for each variable in eqn (1), the number of experiments become prohibitively large and impractical. Therefore, only the most important non-dimensional groups from the above equation are considered. By summarizing eqn (1) we will have:

$$
\frac{\dot{m}_{\text {inf }}}{\dot{m}_{\text {tot }}}=f\left(\frac{H}{w_{D A G}}, \alpha, \beta, \operatorname{Re}, \frac{\dot{m}_{B P}}{\dot{m}_{\text {tot }}}\right) \times\left(\frac{T_{D A G}}{T_{R m}}\right)^{A} \times(R . H .)^{B} .
$$
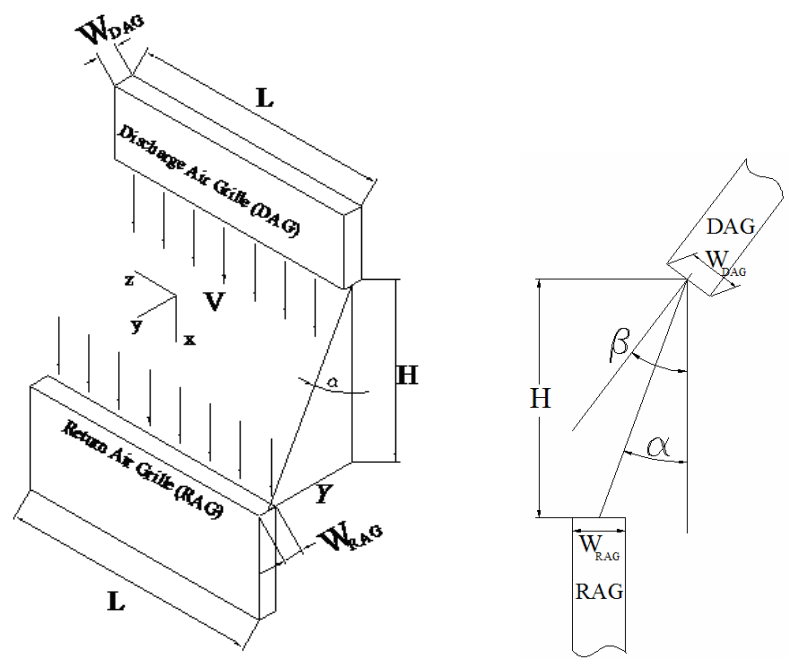

Figure 2: $\quad$ Position of the $R A G$ relative to the $D A G$.

The effects of temperatures and relative humidity are presented by a power law with $A$ and $B$ representing the curve fit constants to be found experimentally. This equation will serve as a guideline to design the modular unit that will be used in our experimental efforts. The overall method can be summarized as: 1) To build a modular air curtain referred to as the proof-of-concept air curtain (POCAC) in which most parameters in eqn (2) can be altered, 2) To measure the infiltration rate directly, 3) To divide the applicable range of each parameter into several intervals and measure the infiltration rate for all permutations, and 4) To use the input and output (infiltration) data for an artificial neural network (ANN) training. The ANN will serve as the tool to not only estimate the infiltration rate of the existing display cases, but also perform parametric studies that will lead to an optimized design. In the current work variables $\frac{H}{w_{D A G}}, \alpha, \beta, \operatorname{Re}, \frac{\dot{m}_{B P}}{\dot{m}_{\text {tot }}}$ take 3 , $3,4,4$, and 4 values between their recommended minimums and maximums, respectively. Therefore, a total of 576 combined numerical and experimental results are required to have a reasonable resolution of data.

Table 1 shows the parameters and their corresponding values in their assigned levels. A matrix of test cases was constructed and the data was collected for about one fourth of the total required number. The results were used to train the 
ANN program. The ANN prediction for known data provided the level of accuracy. Then the resolution of data was increased by increments of 10-20 experiments until the required accuracy was achieved.

Table 1: $\quad$ Independent variables and their values.

\begin{tabular}{|c|c|c|c|c|}
\hline$\alpha$ (degree) & 0 & 16 & 24 & \\
\hline$H / w$ & 8 & 12 & 16 & \\
\hline$\dot{m}_{B P} / \dot{m}_{t o t}$ & \multicolumn{3}{|c|}{ (average values $)=0,0.35,0.55,1$} & \\
\hline$\beta($ degree $)$ & -5 & 0 & 5 & 13 \\
\hline$R e_{R A G}$ & \multicolumn{4}{|c|}{4 values for each "Back Panel flow" ratios, ranging from about 2200 to 12300} \\
\hline
\end{tabular}

\section{Experimental set up}

The modular display case (Figure 3) was constructed such that all the variables in eqn (2) can be easily varied. The installed horizontal and vertical bellows, help changing the $Y$ and $H$, or $\alpha$ and $\frac{H}{w_{D A G}}$, respectively. Furthermore, by using the vertical bellows the discharge duct can rotate to provide the desired throw angle $(\beta)$. In addition, an attempt was made to lower the turbulence intensity at the discharge of the air curtain to minimize the effect of the turbulence intensity at the jet origination point on the infiltration rate. Thus, to lower the turbulence intensity several flow straightners were inserted inside the air passages. The air flow was supplied by a cross-flow fan that spanned over the entire length $(L)$ of the display case and the velocity of the flow at the discharged was controlled by a DC frequency controller integrated to the fan. The length $(L)$ of the POCAC is $114 \mathrm{~cm}$ and the width of the $D A G$ and $R A G$ are respectively $4.1 \mathrm{~cm}$.

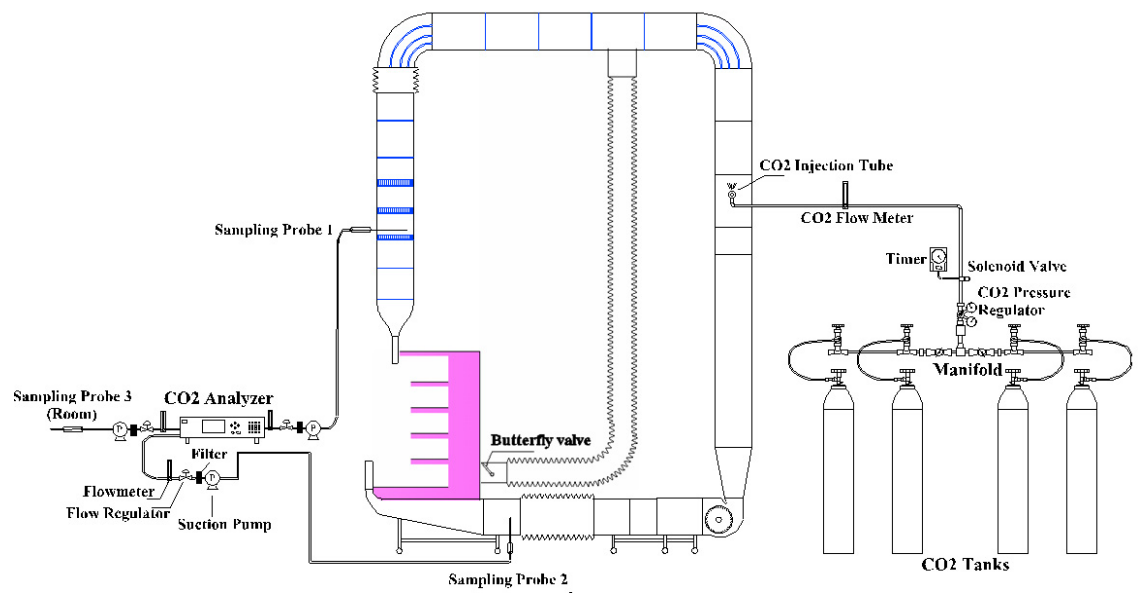

Figure 3: Experimental set up. 


\section{Infiltration measurement}

The conventional experimental method for measuring the infiltration rate in an open refrigerated display case is based on weighting the condensate collected from the cooling coils. Later on, a methodology based on the enthalpy of the room, DAG, and overspill air was developed [11].

However, both methods in an isothermal problem will fail. We have used a tracer gas method to directly monitor the footprint of the infiltrated air through the concentration measurements. In this application, a tracer gas is continuously injected in the upstream and sufficiently far from the $D A G$ after the fan. A portion of the tracer gas will reach the $R A G$ after it is mixed with outside air and the remainder is spilled into a large room. The concentration of the tracer gas is monitored inside the $R A G$ duct (before the fan) and the room. Figure 3 illustrates the measurement equipment and sampling locations. To provide air (including the tracer gas) to the perforated back panel, 3 ducts were used after the injection point of the tracer gas to branch off a portion of the total flow towards the back panel. The flow rate through the perforated back panel was varied by valves. Amin et al [18] have analytically demonstrated that a relationship between the tracer gas concentrations and the infiltration rate can be established as:

$$
\dot{m}_{\text {inf }}=\dot{m}_{R A G} \times\left|\frac{C_{D A G}-C_{R A G}}{C_{D A G}-C_{R m}}\right| .
$$

This equation indicates that the absolute amount of infiltrated mass depends on the concentration of the tracer gas wherever it exists and the total flow rate of the display case, i.e. the $R A G$ flow rate. Therefore, it is required to measure the concentration of the tracer gas at three locations: 1) upstream of the $D A G$ after the injection point, 2) downstream of the $R A G$ before the fan and injection point, and 3 ) the room. To express the infiltration rate as a non-dimensional scalable quantity and also to make the output compatible with the ANN algorithm (all variables must be between 0 and 1) a non-dimensional Infiltration Rate (N.I.R.) can be written as in eqn (4).

$$
\text { N.I.R. }=\frac{\dot{m}_{\mathrm{inf}}}{\dot{m}_{R A G}}=\left|\frac{C_{D A G}-C_{R A G}}{C_{D A G}-C_{R m}}\right| .
$$

This equation resembles the thermal entrainment used in some previous studies [19] given by:

$$
\eta=\left|\frac{T_{D A G}-T_{R A G}}{T_{D A G}-T_{R m}}\right| .
$$

In the limiting case, when the entire discharged tracer gas returns to the return duct $\left(C_{D A G}=C_{R A G}\right.$ in eqn (4)) the infiltration goes to zero, that is consistent with $T_{D A G}=T_{R A G}$ and $\eta \rightarrow 0$ in eqn (5). Similarly if no portion of the air from the $D A G$ reaches the $R A G$ (for instance when the air curtain breaks due to bending outward), the $R A G$ concentration and temperature become identical to that of the room assuming that the room is a large reservoir. Therefore, if $C_{R m}=C_{R A G}$ or similarly $T_{R m}=T_{R A G}$, they will yield equivalent results, i.e. N.I.R. $\rightarrow 1$ and $\eta \rightarrow 1$. 


\section{Results}

The velocity profile at the $D A G$ for the POCAC was measured and visualized by the DPIV method. The desired Top-Hat profile was observed and a turbulence intensity of about $2 \%$ was measured. Figure 4 shows the velocity profile at different Reynolds numbers. It is shown [12] that the Top-Hat profile is the best practical profile that can exist at the $D A G$ for reducing the infiltration rate. Carbon dioxide $\left(\mathrm{CO}_{2}\right)$ has been used as the tracer gas in the current work. Its density is comparable to that of air and it is colorless, odorless, non-corrosive, not flammable, and not hazardous for up to 5000 ppm (for 8 hours of continuous exposure in the room) which is about 15 times more than its amount in nature (350 ppm). The maximum amount of the injected $\mathrm{CO}_{2}$ is about $25000 \mathrm{ppm}$ (equivalent to $2.5 \%$ mass fraction) before the $D A G$ that falls under $5000 \mathrm{ppm}$ $(0.5 \%)$ in the room and across the air curtain. Figure 5a shows the concentration of the tracer gas at the three points of interest in a typical experiment including the N.I.R. It can be seen that the N.I.R. remains constant even after the injection of the tracer gas has stopped. The results are for steady state operation of the display case and air curtain.

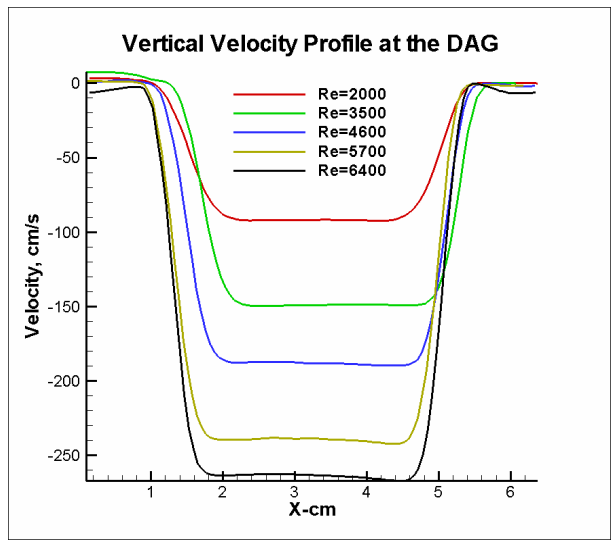

Figure 4: $\quad$ Velocity profile at the $D A G$ of the experimental set up.

This is expected, because both concentrations at $D A G$ and $R A G$ are being reduced almost at the same rate. Figure $5 \mathrm{~b}$ shows the absolute infiltration as a function of the opening height while other parameters are maintained the same. It is seen that for this case a maximum point exists at which the infiltration increases. The 2D numerical simulation for several configurations was performed by the Fluent code [20]. The simulation was performed with $\mathrm{CO}_{2}$ as the tracer gas and validated for several test cases. Then the numerical simulation was used to increase the resolution of the required data points for the ANN, when needed. Figures $6 \mathrm{a} \& 6 \mathrm{~b}$ exhibit the streamlines and mass concentration of $\mathrm{CO}_{2}$ as predicted by the CFD simulation. No mass flow boundary condition was specified at the $D A G$. Instead a fan boundary condition was assigned at the inner duct of the POCAC that yields the experimental flow rate. The CFD results also 
help us with identification of the regions in the room concerning the tracer gas sampling locations. In addition, Figure 6 a indicates that the entrainment of room air occurs almost across the entire opening height and indicates that the room $\mathrm{CO}_{2}$ is the highest near the floor.
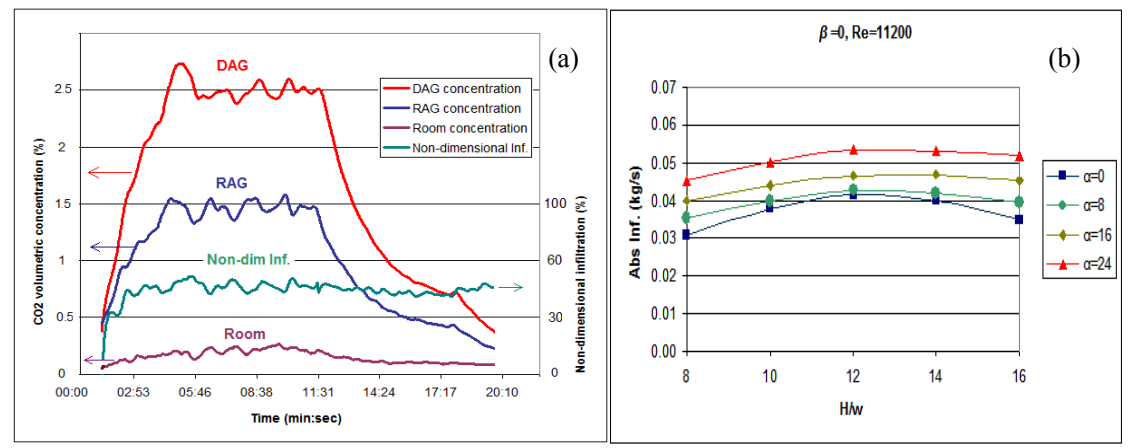

Figure 5: Experimental results: a) tracer gas concentrations and nondimensional infiltration; b) absolute infiltration rates.
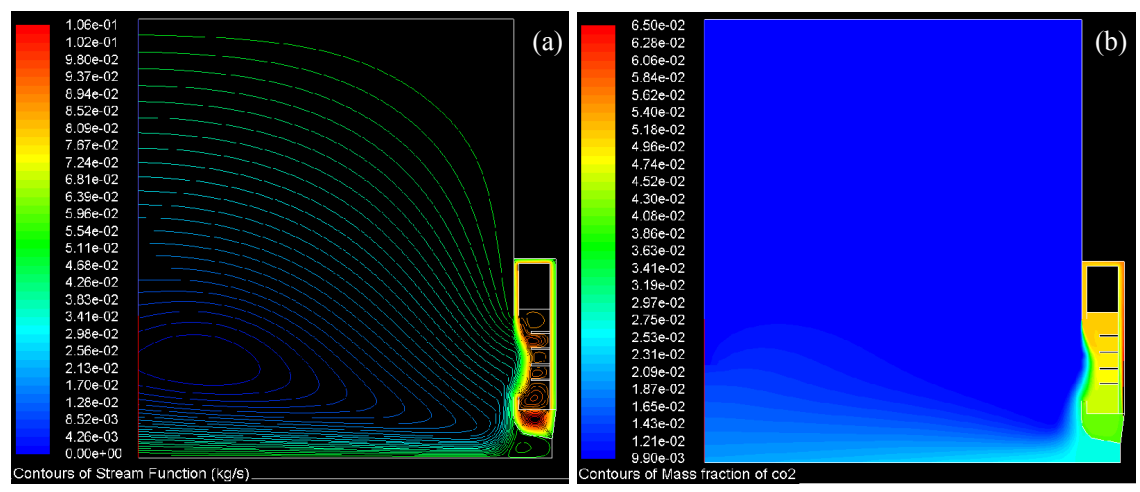

Figure 6: CFD results: a) streamline of flow inside and outside the POCAC; b) mass concentration of $\mathrm{CO}_{2}$.

Hence positioning of the sampling probe close to the floor will produce false information regarding the room $\mathrm{CO}_{2}$ concentration. After the completion of all tests, ANN is used as a practical tool to predict the infiltration rate for any other display case as well as conducting parametric studies. Artificial neural networks (ANNs) in fact provide an alternative paradigm or mechanism for performing regression or curve fitting as compared to classical regression methods. In other words, ANNs denote a tool that achieves an implementation of regression. The classical regression approaches are linear whereas ANN is a generalization of regression to non-linear systems. A sample of the ANN prediction after its proper training with actual data is shown in Table 2. As it is seen from this table, an excellent agreement between the prediction and actual data exists. It is evident 
that the accuracy of ANN prediction relies on the number of available data points (resolution).

Table 2: Comparison of the N.I.R. of experimental data with ANN predictions.

\begin{tabular}{|c|c|c|c|c||c|c|}
\hline$\alpha\left({ }^{\circ}\right)$ & $\beta\left({ }^{\circ}\right)$ & $\operatorname{Re}_{D A G}$ & $\dot{m}_{B P} / \dot{m}_{\text {tot }}$ & $H / w_{D A G}$ & \multicolumn{2}{|c|}{$\begin{array}{c}\text { Non-dimensional } \\
\text { Infiltration Rate }\end{array}$} \\
\hline \hline 0 & -5 & 5500 & 0 & 8 & Actual & ANN \\
\hline 0 & 0 & 5500 & 0.37 & 12 & 0.239 & 0.244 \\
\hline 0 & 13 & 3400 & 0.46 & 8 & 0.302 & 0.293 \\
\hline 16 & 0 & 3400 & 0.54 & 16 & 0.270 & 0.281 \\
\hline 16 & -5 & 8400 & 0.41 & 16 & 0.295 & 0.332 \\
\hline 16 & 13 & 8400 & 0.41 & 8 & 0.329 & 0.320 \\
\hline 24 & -5 & 8400 & 0 & 12 & 0.211 & 0.199 \\
\hline 24 & 5 & 2200 & 0.34 & 12 & 0.424 & 0.395 \\
\hline 24 & 13 & 3400 & 1 & 16 & 0.419 & 0.410 \\
\hline
\end{tabular}

\section{Conclusion}

A systematic approach for solving a large scale problem with many variables is suggested. The solution method relies on hybrid experimental/numerical methods and develops a practical tool based on the collected data and artificial neural network algorithm. The accuracy of results indicates the feasibility of the approach and its versatility to be applied to the problems of the same magnitude.

\section{Acknowledgements}

This work was partially sponsored by the US Department of Energy, Office of Building Technology, State and Community Programs under contract DE-AC0500OR22725 with UT-Battelle, LLC. The authors wish to thank Southern California Edison CO. for allowing us to use their RTTC facilities for our testing and also Mr. Van D. Baxter from The Oakridge National Laboratory for monitoring the project and providing us with his support and advice.

\section{References}

[1] Howell, R.H., Van, N. \& Smith, C.E., Heat and Moisture Transfer through Recirculated Plane Air Curtains. ASHRAE Trans., 82(1), pp. 191-205, 1976.

[2] Howell, R.H. \& Shiabata, M., Optimum Heat Transfer through Turbulent Recirculated Plane Air Curtains. ASHRAE Trans., 86(1), pp. 188-200, 1980.

[3] Van N. \& Howell, R.H., Influence of Initial Turbulence Intensity on the Development of Plane Air Curtain Jets. ASHRAE Trans., 82(1), pp. 208$228,1983$. 
[4] Rouaud, O. \& Havet, M., Behavior of an Air Curtain Subjected to Transversal Pressure Variations. J. of Env. Eng. 132(2), pp. 263-270, 2006.

[5] Hayes, F.C. \& Stoecker, W.F., Design Data for Air Curtains. ASHRAE Trans., No 2121, pp. 153-167, 1969.

[6] Howell, R.H. \& Adams, P.A., Effects of Indoor Space Conditions on Refrigerated Display Case Performance. ASHRAE 596RP, Department of Mechanical Engineering, University of South Florida, Tampa, Florida, November 1991.

[7] Stribling, D., Tassou, S.A. \& Mariott D. A Two-Dimensional CFD Model of a Refrigerated Display Case. ASHRAE Transaction, 104, pp. 88-94, 1997.

[8] Axell, M. \& Fahlen P., Vertical Display Cabinet. SP (The Swedish National Testing and Research Institute): Sweden, 2002.

[9] Axell, M. \& Fahlen P., Design Criteria for Energy Efficient Vertical Air Curtains in Display Cabinets. International Congress of Refrigeration: Washington, DC., 2003.

[10] Navaz, H.K., Faramarzi, R., Dabiri, D., Gharib, M. \& Modarress, D., The Application of Advanced Methods in Analyzing the Performance of the Air Curtain in a Refrigerated Display Case. J. of Fluid Eng., 124(3), pp. 756764, 2002.

[11] Navaz, H.K., Henderson, B.S., Faramarzi, R., Pourmovahed, A. \& Taugwalder, F., Jet Entrainment Rate in Air Curtain of Open Refrigerated Display Cases. Int. J. of Refrigeration, 28(2), pp. 265-275, 2005.

[12] Navaz, H.K., Amin, M., Srinivasan C.R. \& Faramarzi, R., Jet Entrainment Minimization in Air Curtain of Open Refrigerated Display Cases, International Journal of Numerical Methods for Heat and Fluid Flow, 16(4), pp. 417-430, 2006.

[13] Navaz, H.K. \& Faramarzi, R., Advanced Supermarket Display Case Workshop. ASHRAE winter meeting: Anaheim, California, January 2004.

[14] Field, B., Kalluri, R. \& Loth, E., PIV Investigation of AIR-Curtain Entrainment in Open Display Cases. IIF-II-Commission D1/B1: Urbana, IL, 2002.

[15] Field, B. \& Loth, E., An Air Curtain Long a Wall with High Inlet Turbulence. Journal of Fluid Engineering, 126, pp. 391-398, 2004.

[16] Chen, Y., Yuaa, X., Simulation of a cavity insulated by a vertical single band cold air curtain. Energy Conv. \& Manag., 46, pp. 1745-1756, 2005.

[17] Mhiri, H., Golli E., S., Berthon, A., Palec, G.L. \& Bournot, P., Numerical study of the thermal and aerodynamic insulation of a cavity with a vertical downstream air jet. Int. Comm. Heat Mass Transfer, 25(7), pp. 919-928, 1998.

[18] Amin, M., Navaz, H.K., Dabiri, D. \& R. Faramarzi, Air Curtain Studies in Vertical Open Refrigerated Display Cases: Minimization of Infiltration Rate. Final Interim Report Prepared for the Southern California Edison CO., 2008.

[19] Field B. \& Loth, E., Entrainment of refrigerated air curtains down a wall. Experimental Thermal and Fluid Science, 30, pp. 175-184, 2006.

[20] The ${ }^{\odot}$ Fluent Code by Fluent, Inc. 